\title{
MEASUREMENT OF THE MASS OF THE W BOSON AT LEP AND DETERMINATION OF ELECTROWEAK PARAMETERS
}

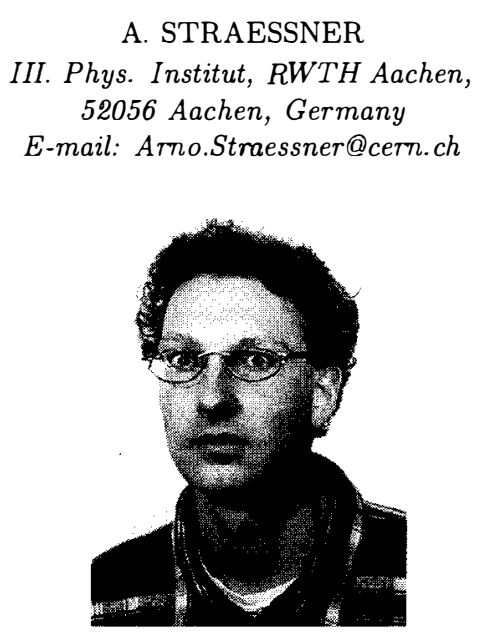

New results of the $\mathrm{W}$ mass and width measurements at LEP are presented, which yield $M_{\mathrm{W}}=80.401 \pm 0.048 \mathrm{GeV}$ and $\Gamma_{\mathrm{W}}=2.19 \pm 0.15 \mathrm{GeV}$. A comparison of this direct and the indirect $\mathrm{W}$ mass, obtained in an analysis of electroweak measurements, is made and good agreement is observed. All electroweak data are very consistent with the Standard Model predictions. In a combined fit an upper limit on the mass of Higgs boson is put to $M_{\mathrm{H}}<188 \mathrm{GeV}$, while direct searches at LEP exclude Higgs masses below $107.9 \mathrm{GeV}$ at $95 \%$ confidence level.

\section{Introduction}

In 1989 the LEP $\mathrm{e}^{+} \mathrm{e}^{-}$collider started operation at centre-of-mass energies close to the $\mathrm{Z}$ resonance. Each of the four LEP experiments, ALEPH, DELPHI, L3 and OPAL, collected about 4 million $\mathrm{Z}$ decays. The data allow a precise determination of the properties of the $\mathrm{Z}$ boson. In the second phase of LEP, starting in 1996, the centre-of-mass energy, $\sqrt{s}$, is increased to energies beyond the production threshold for $\cdot \mathrm{W}$ boson pairs up to $\sqrt{s}=202 \mathrm{GeV}$ in 1999 . About $7000 \mathrm{~W}$ pair events per experiment are used to measure the mass and the width of the $\mathrm{W}$ boson. The data corresponds to an integrated luminosity of about $4 \times 480 \mathrm{pb}^{-1}$.

The properties of the heavy gauge bosons, $\mathrm{Z}$ and $\mathrm{W}$, are important quantities in the Standard Model (SM). Combining the LEP results with other electroweak measurements stringent tests of the SM predictions are performed. A constraint is put on the mass of the last unknown particle in the SM: the Higgs boson. 

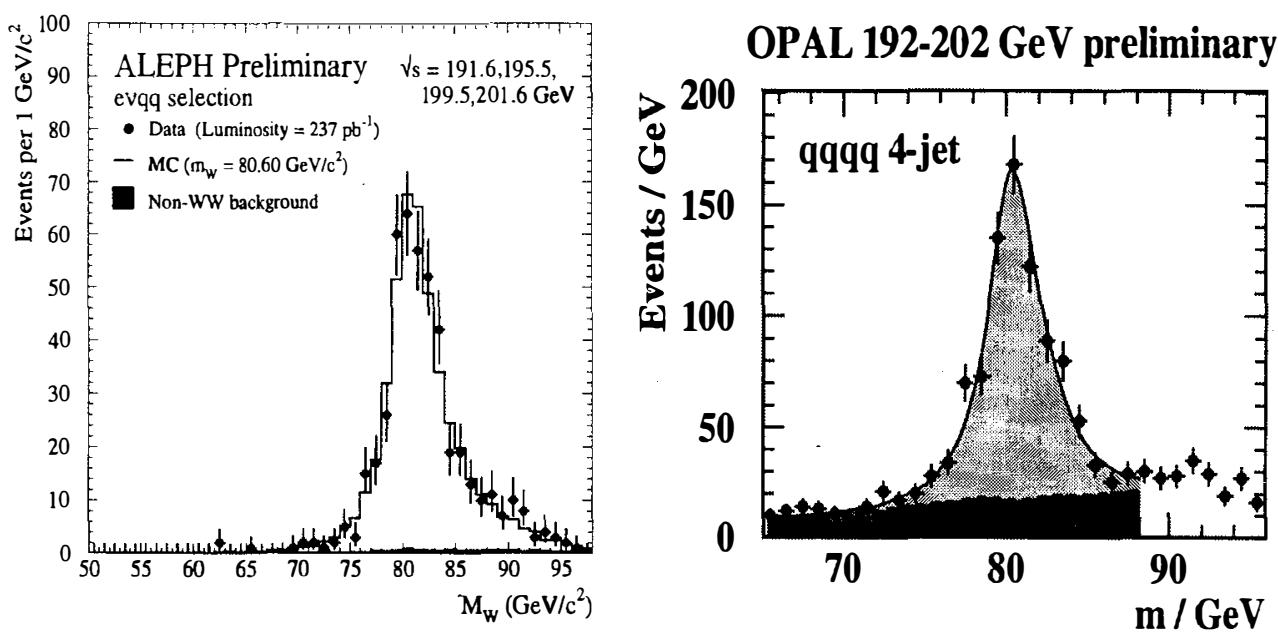

Figure 1: Invariant mass spectra measured in qqe $\nu$ and qqqq events at $\sqrt{s}=192-202 \mathrm{GeV}$ compared to the W mass fit results. For the preliminary results of 1999 the OPAL collaboration uses a relativistic Breit-Wigner function instead of reweighted Monte Carlo samples for comparison with data, as it is shown on the right.

\section{W Mass Measurement at LEP}

$\mathrm{W}$ bosons are produced at LEP in the reaction $\mathrm{e}^{+} \mathrm{e}^{-} \rightarrow \mathrm{WW}$ at centre-of-mass energies between $161 \mathrm{GeV}$ and $202 \mathrm{GeV}$. At threshold the total cross-section for W-pair production, $\sigma_{\mathrm{Ww}}$, is strongly dependent on the mass of the W boson. From measurements of $\sigma_{\mathrm{Ww}}$ at $\sqrt{s}=161-172 \mathrm{GeV}$ a value of $M_{\mathrm{W}}=80.40 \pm 0.22 \mathrm{GeV}$ is derived ${ }^{1}$. At higher energies the sensitivity of $\sigma_{\mathrm{Ww}}$ on $M_{\mathrm{W}}$ is less pronounced. Therefore an extraction method based on the direct reconstruction of the $\mathrm{W}$ decay products is applied.

The two $\mathrm{W}$ bosons of each event decay either to a pair of quarks, e.g. $\mathrm{W}^{-} \rightarrow \overline{\mathrm{u}} \mathrm{d}$ or $\overline{\mathrm{c}} \mathrm{s}$, or to a lepton and a neutrino, $\mathrm{W}^{-} \rightarrow \ell^{-} \bar{\nu}_{\ell},(\ell=\mathrm{e}, \mu, \tau)$; in the following denoted qq and $\ell \nu$ for short. This leads to three possible final states, qqqq, qq $\ell \nu$, and $\ell \nu \ell \nu$. Because of the two neutrinos in the $\ell \nu \ell \nu$ final state it is more difficult to reconstruct the complete event topology. For a $\mathrm{W}$ mass measurement the fully hadronic and the semi-leptonic decays are therefore more important, which have a branching fraction of $45 \%$ and $44 \%$, respectively.

Kinematic constraints are imposed to improve the resolution of the measured fermion energies and angles. In a kinematic fit these quantities are varied within their experimental resolution to satisfy energy and momentum conservation. As a possible additional constraint the two reconstructed $\mathrm{W}$ masses are forced to be equal.

The mass of the $\mathrm{W}$ boson is extracted from the measured invariant masses by comparing Monte Carlo event samples to data. Samples for different values of $M_{\mathrm{W}}$ are obtained in an event reweighting procedure. Unbinned maximum likelihood fits to the measured spectra are performed in the qq $\ell \nu$ and qqqq channels, as it is illustrated in figure 1 . In fully hadronic events also hard gluon radiation from quarks is taken into account. By splitting the events into a 4 -jet and a 5 -jet sample the overall mass resolution is improved ${ }^{2}$. The DELPHI collaboration uses a convolution method for extracting the $\mathrm{W}$ mass ${ }^{2}$. With the current methods the $\mathrm{W}$ mass is measured at LEP with a statistical precision of $27 \mathrm{MeV}$ combining the direct reconstruction measurements performed at $172-202 \mathrm{GeV}$.

A systematic error common to all measurements comes from the uncertainty on the LEP 
beam energy, which amounts to $20-21 \mathrm{MeV}$ for the highest beam energies ${ }^{3}$. Since it is a relative error the corresponding uncertainty on $M_{\mathrm{W}}$ is $17 \mathrm{MeV}$. In 1999 a new beam spectrometer is installed in LEP which is expected to be fully operational in the running period 2000. With this device the LEP energy error can be reduced to $10-15 \mathrm{MeV}^{4}$.

A particular problem of the $\mathrm{W}$ mass measurement appears in the fully hadronic decay channel. The distance between the two decaying $\mathrm{W}$ bosons is about $0.1 \mathrm{fm}$, which is much smaller than the hadronic interaction length of typically $1 \mathrm{fm}$. This can give rise to hadronic final state interactions (FSI), such as colour reconnection effects ${ }^{5}$ or Bose-Einstein correlations ${ }^{6}$. Both effects lead to momentum transfer between particles coming from different $\mathrm{W}$ bosons, which affects the reconstruction of the invariant masses. By studying various FSI models the possible systematic bias is estimated to be $52 \mathrm{MeV}$, which is large compared to a statistical error of only $37 \mathrm{MeV}$ in the qqqq channel. FSI effects may also show up in the difference between the semi-leptonic and the fully hadronic $\mathrm{W}$ mass:

$$
\Delta M_{\mathrm{W}}=M_{\mathrm{W}}(\mathrm{qqqq})-M_{\mathrm{W}}(\text { non-qqqq })=35 \pm 55 \mathrm{MeV},
$$

where systematic errors due to possible FSI effects are removed. However, the observed value is compatible with zero. Possible effects of FSI are also studied most recently in other observables than the $\mathrm{W}$ mass which are sensitive to $\mathrm{FSI}^{7}$, e.g. the particle flow in the overlap region between two jets and particle correlation functions. In future it may be possible to excluded some of the FSI models in a combined LEP analysis, which should reduce the systematic uncertainty in $M_{\mathrm{W}}$.

Table 1 summarises the mass values measured by the LEP experiments in non-qqqq and qqqq W-pair events using the direct reconstruction method ${ }^{2}$. All values agree well within the given errors. The combined $\mathrm{W}$ mass measurement split up into non-qqqq and qqqq channels yields

$$
\begin{array}{ll}
M_{\mathrm{W}} \text { (non-qqqq) } & =80.398 \pm 0.039 \text { (stat.) } \pm 0.031 \text { (syst.) } \pm 0.017 \text { (LEP) GeV } \\
M_{\mathrm{W}} \text { (qqqq) } & =80.408 \pm 0.037 \text { (stat.) } \pm 0.031 \text { (syst.) } \pm 0.052 \text { (FSI) } \pm 0.017 \text { (LEP) GeV }
\end{array}
$$

where the two masses are $31 \%$ correlated. The combined mass value for all channels is

$$
M_{\mathrm{W}}(\mathrm{fff})=80.401 \pm 0.027 \text { (stat.) } \pm 0.031 \text { (syst.) } \pm 0.018 \text { (FSI) } \pm 0.017 \text { (LEP) GeV, }
$$

with a good $\chi^{2}$ of 30.7 for 27 degrees of freedom. Apart from errors due to FSI and the LEP beam energy, the dominant systematic error originates from different fragmentation models: Including the result derived from the cross-section measurements yields finally ${ }^{1}$ :

$$
M_{\mathrm{W}}^{\mathrm{LEP}}=80.401 \pm 0.048 \mathrm{GeV} .
$$

Other direct $\mathrm{W}$ mass measurements performed at $\mathrm{p} \overline{\mathrm{p}}$-colliders ${ }^{8}$ are in very good agreement with this result. The combination of all direct $\mathrm{W}$ mass determinations results in ${ }^{9}$

$$
M_{\mathrm{W}}^{\mathrm{LEP}+\mathrm{p} \overline{\mathrm{p}}}=80.419 \pm 0.038 \mathrm{GeV} .
$$

At LEP also the width of the $\mathrm{W}$ boson, $\Gamma_{\mathrm{W}}$, is measured using the direct reconstruction method. The results are summarised in table 1 : The LEP combined value is ${ }^{1}$

$$
\Gamma_{\mathrm{W}}^{\mathrm{LEP}}=2.19 \pm 0.15 \mathrm{GeV} .
$$

This result shows good agreement with the direct measurement at the Tevatron by the CDF collaboration which yields $\Gamma_{\mathrm{W}}=2.06 \pm 0.13 \mathrm{GeV}^{10}$. It also agrees well with the $\mathrm{SM}$ prediction of $\Gamma_{\mathrm{W}}=2.08 \mathrm{GeV}$ using the current world average $\mathrm{W}$ mass ${ }^{11}$. 
Table 1: W mass and width measurement by direct reconstruction from the four LEP experiments. The mass values are given separately for the non-qqqq qqqq and ffff channels. The errors include statistics and systematics. The procedure to obtain the combined LEP mass values treats correlations between systematic errors in a slighlty different way than the individual experiments. Therefore the LEP average in the last row is not identical with the average of the values shown in the upper four rows. All mass numbers contain the $172-202 \mathrm{GeV}$ data, except the DELPHI result which is based on the $172-189 \mathrm{GeV}$ data. Also the width is analysed by the LEP experiments only in a restricted data set.

\begin{tabular}{|c||c|c|c|c|}
\hline \multicolumn{1}{|c||}{ Experiment } & \multicolumn{3}{|c|}{$M_{\mathrm{W}}[\mathrm{GeV}]$} & $\Gamma_{\mathrm{w}}[\mathrm{GeV}]$ \\
& non-qqqq & qqqq & ffff & ffff \\
\hline Aleph & $80.435 \pm 0.079$ & $80.467 \pm 0.086$ & $80.449 \pm 0.065$ & $2.17 \pm 0.20$ \\
Delphi & $80.230 \pm 0.140$ & $80.360 \pm 0.115$ & $80.308 \pm 0.090$ & $2.48 \pm 0.41$ \\
L3 & $80.282 \pm 0.102$ & $80.489 \pm 0.132$ & $80.353 \pm 0.088$ & $2.18 \pm 0.22$ \\
Opal & $80.483 \pm 0.078$ & $80.380 \pm 0.103$ & $80.446 \pm 0.064$ & $1.86 \pm 0.37$ \\
\hline LEP & $80.398 \pm 0.053$ & $80.408 \pm 0.074$ & $80.401 \pm 0.049$ & $2.19 \pm 0.15$ \\
\hline
\end{tabular}

\section{Determination of Electroweak Parameters}

\subsection{Input Parameters to the Standard Model}

Electroweak observables can be computed in the SM framework as a function of a few parameters: the fine structure constant, $\alpha$, the strong coupling constant, $\alpha_{s}$, the $\mathrm{Z}$ mass, $M_{\mathrm{Z}}$, the W mass, $M_{\mathrm{W}}$, the top quark mass, $M_{\mathrm{t}}$, the Higgs boson mass, $M_{\mathrm{H}}$, and the Cabbibo-KobayashiMaskawa mixing matrix, $V_{\mathrm{CKM}}$.

The $\mathrm{W}$ mass is currently measured with a relative precision of $0.05 \%$. Since the direct measurements of $M_{\mathrm{W}}$ are available only recently the $\mathrm{W}$ mass is not used as input parameter but it is replaced by the more precise muon decay constant, $G_{\mathrm{F}}=(1.16639 \pm 0.0002) \cdot 10^{-5} \mathrm{GeV}^{-2}$. The $\mathrm{W}$ mass is then a derived quantity, to be compared with the measurement.

For the calculation of observables measured at the $\mathrm{Z}$ pole the running of the coupling constant $\alpha$ with energy scale $Q^{2}$ has to be taken into account. At $Q^{2}=M_{\mathrm{Z}}^{2}$ its value is $\alpha\left(M_{\mathrm{Z}}^{2}\right)^{-1}=128.878 \pm 0.090^{12}$. The largest part of the error originates from uncertainties on the contributions from the five lightest quarks to the photon vacuum polarisation, parametrised by $\Delta \alpha_{\text {had. }}^{(5)}$. The value of $\Delta \alpha_{\text {had }}^{(5)}=0.02804 \pm 0.00065$ is derived from an analysis of measurements of the cross-section $\mathrm{e}^{+} \mathrm{e}^{-} \rightarrow$ hadrons below the $\mathrm{Z}$ resonance. The accuracy of only $2 \%$ limits the precision of the SM predictions. There are other estimations of this quantity which have a smaller uncertainty because they are more theory driven, e.g. that of reference 13 which is used for comparison.

The set of free parameters which are varied in the SM fit consists of $\Delta \alpha_{\text {had }}^{(5)}, \alpha_{s}\left(M_{\mathrm{Z}}^{2}\right), M_{\mathrm{Z}}, M_{\mathrm{t}}$, and $M_{\mathrm{H}}$. These parameters are constrained by the measurements of electroweak observables.

\subsection{Electroweak Measurements}

Here, a short overview of the results of the electroweak measurements is given. The combination of the measured electroweak observables at LEP and SLC is summarised in reference 9, which also contains further details about the interpretation of the results within the SM. 
Table 2: Average line shape parameters measured at LEP. Values without and with assumption of lepton universality are given in the second and third column, respectively.

\begin{tabular}{|r|c|c|}
\hline Parameter & $\begin{array}{c}\text { without } \\
\text { lepton universality }\end{array}$ & $\begin{array}{c}\text { with } \\
\text { lepton universality }\end{array}$ \\
\hline$M_{\mathrm{Z}}[\mathrm{GeV}]$ & $91.1872 \pm 0.0021$ & $91.1871 \pm 0.0021$ \\
$\Gamma_{\mathrm{Z}}[\mathrm{GeV}]$ & $2.4944 \pm 0.0024$ & $2.4944 \pm 0.0024$ \\
$\sigma_{\text {had }}^{0}[\mathrm{nb}]$ & $41.544 \pm 0.037$ & $41.544 \pm 0.037$ \\
$R_{\mathrm{e}}$ & $20.803 \pm 0.049$ & - \\
$R_{\mu}$ & $20.786 \pm 0.033$ & - \\
$R_{\tau}$ & $20.764 \pm 0.045$ & - \\
\hline$R_{\ell}$ & - & $20.768 \pm 0.024$ \\
\hline
\end{tabular}

Results from $Z$ line shape at LEP

The shape of the $\mathrm{Z}$ resonance is characterised by three parameters, the $\mathrm{Z}$ mass, $M_{\mathrm{Z}}$, the width, $\Gamma_{\mathbf{Z}}$, and the height of the resonance as given by the pole cross-section, $\sigma_{\mathrm{ff}}^{0}$ :

$$
\sigma_{\mathrm{ff}}^{0}=\frac{12 \pi}{M_{\mathrm{Z}}^{2}} \frac{\Gamma_{\mathrm{ee}} \Gamma_{\mathrm{ff}}}{\Gamma_{\mathrm{Z}}} .
$$

The separate measurements of $Z$ decays for different lepton flavours allow the determination of the corresponding partial decay widths of the $\mathrm{Z}$, which are given by the inverse ratio to the hadronic decay width, $R_{\ell}=\Gamma_{\text {had }} / \Gamma_{\ell \ell},(\ell=\mathrm{e}, \mu, \tau)$. The average line shape parameters of the four LEP experiments are shown in table 2. The measurements of $R_{\ell}$ for the three lepton species prove the lepton universality of the neutral weak interaction.

The $\mathrm{Z}$ boson can decay also invisibly into neutrino pairs. From the invisible decay width, $\Gamma_{\text {inv }} / \Gamma_{\ell \ell}=5.941 \pm 0.016$, and the SM calculation for $\Gamma_{\nu \nu}$ the number of neutrino species is determined to be $N_{\nu}=2.9835 \pm 0.0083$, which is two standard deviations below the expected value of 3 .

\section{Heavy Flavour Results}

Experimental techniques to tag the quark flavour in hadronic decays make it possible to measure also the $\mathrm{Z}$ decay width into the heavy quarks, $\mathrm{b}$ and $\mathrm{c}$. The results are presented in terms of the ratios

$$
R_{\mathrm{b}}=\frac{\Gamma_{\mathrm{b} \overline{\mathrm{b}}}}{\Gamma_{\mathrm{had}}}, \quad R_{\mathrm{c}}=\frac{\Gamma_{\mathrm{c} \overline{\mathrm{c}}}}{\Gamma_{\mathrm{had}}} .
$$

With this definition most of the radiative corrections cancel out, except the important corrections from the $\mathrm{Zb} \bar{b}$ vertex. In the $\mathrm{SM}$ this contribution basically depends only on a single parameter which is the top quark mass. The combined analysis of the LEP and SLD data yields

$$
R_{\mathrm{b}}=0.21642 \pm 0.00073 \quad R_{\mathrm{c}}=0.1674 \pm 0.0038,
$$

with a negative correlation of $14 \%$ between the two quantities.

\section{Asymmetry Measurements}

Parity violation in the neutral current $\mathrm{Z}$ decays is caused by the different couplings to lefthanded and right-handed fermions. With the definition of the asymmetry parameter

$$
\mathcal{A}_{\mathrm{f}}=\frac{2 g_{\mathrm{V}}^{\mathrm{f}} g_{\mathrm{A}}^{\mathrm{f}}}{\left(g_{\mathrm{V}}^{\mathrm{f}}\right)^{2}+\left(g_{\mathrm{A}}^{\mathrm{f}}\right)^{2}}
$$


where $g_{\mathrm{V}}^{\mathrm{f}}$ and $g_{\mathrm{A}}^{\mathrm{f}}$ are the effective vector and axial vector coupling constants, one can write the $\mathrm{Z}$ forward-backward asymmetries and the tau polarisation in terms of $\mathcal{A}_{\mathrm{f}}$ :

$$
A_{\mathrm{FB}}^{0, \mathrm{f}}=\frac{3}{4} \mathcal{A}_{\mathrm{e}} \mathcal{A}_{\mathrm{f}}, \quad \mathcal{P}_{\tau}(\cos \theta)=-\frac{\mathcal{A}_{\tau}\left(1+\cos ^{2} \theta\right)+2 \mathcal{A}_{\mathrm{e}} \cos \theta}{1+\cos ^{2} \theta+2 \mathcal{A}_{\tau} \mathcal{A}_{\mathrm{e}} \cos \theta} .
$$

Forward-backward asymmetries are measured separately for the leptonic channels, for $\mathrm{b}$ and $\mathrm{c}$ quarks, and inclusively for hadronic decays in terms of the charge asymmetry, $\left\langle Q_{\mathrm{FB}}\right\rangle$. From the tau polarisation the values of $\mathcal{A}_{\mathrm{e}}$ and $\mathcal{A}_{\tau}$ are extracted.

At SLD the incoming electron beam is longitudinally polarised which allows the determination also of the left-right asymmetries, $A_{\mathrm{LR}}^{0}=\mathcal{A}_{\mathrm{e}}$. In addition, the left-right forward-backward asymmetries have been measured for leptons, b and c quarks. From these the parameters $\mathcal{A}_{\ell}$, $\mathcal{A}_{\mathrm{b}}$ and $\mathcal{A}_{\mathrm{c}}$ are determined. The leptonic SLD measurements are usually summarized in terms of $\mathcal{A}_{\ell}$, assuming lepton universality. The updated value of $\mathcal{A}_{\ell}{ }^{14}$ is used in the SM fits.

To compare the different asymmetry measurements it is conventional to use the leptonic effective weak mixing angle, as defined by:

$$
\sin ^{2} \theta_{\mathrm{eff}}^{\text {lept }}=\frac{1}{4}\left(1-\frac{g_{\mathrm{V}}^{\ell}}{g_{\mathrm{A}}^{\ell}}\right)
$$

Figure 2 a shows the results of the LEP and SLD measurements of $\sin ^{2} \theta_{\text {eff }}^{\text {lept }}$, which differ by 2.8 standard deviations. The relative high LEP average is dominated by the quark asymmetry measurements which are more precise than the leptonic asymmetries. On the bottom of figure $2 \mathrm{a}$ the average value is compared to the SM calculations of $\sin ^{2} \theta_{\text {eff }}^{\text {lept }}$ for different Higgs masses; indicating a tendency towards low values of $M_{\mathrm{H}}$.

\section{Other Measurements}

Important constraints come from the direct measurements of $M_{\mathrm{W}}$ at LEP and of $M_{\mathrm{W}}$ and $M_{\mathrm{t}}=174.3 \pm 5.1 \mathrm{GeV}$ at the Tevatron ${ }^{15}$. The result for the on-shell value of $\sin ^{2} \theta_{\mathrm{w}}=$ $0.2255 \pm 0.0021$ measured by NuTeV/CCFR in $\nu \mathrm{N}$ scattering ${ }^{16}$ is also used as input to the fit for the SM parameters.

\subsection{Fits to Electroweak Data}

The electroweak measurements are used to derive the basic SM parameters ${ }^{9}$. The calculation of the SM predictions are performed with the ZFITTER and TOPAZ0 program packages which contain the most recent calculations of higher order radiative corrections. All measurements that are input to the fit are summarized in figure $2 \mathrm{~b}$. Also shown is their difference to the SM fit result relative to the measurement error. All experimental values agree with the predictions.

The resulting SM parameters are listed in table 3 based on different data sets. Interesting comparisons are the direct and indirect determinations of $M_{\mathrm{t}}$ and $M_{\mathrm{W}}$. The top quarks mass derived only from LEP data is $M_{\mathrm{t}}=176_{-11}^{+14} \mathrm{GeV}$, which is in very good agreement with the direct measurement at the Tevatron. Also the indirect $\mathrm{W}$ mass, $M_{\mathrm{W}}^{\mathrm{fit}}=80.382 \pm 0.026 \mathrm{GeV}$ extracted from all electroweak data except the direct $M_{\mathrm{W}}$ measurement is perfectly consistent with $M_{\mathrm{W}}^{\mathrm{LEP}+\mathrm{pp}}=80.419 \pm 0.038 \mathrm{GeV}$. This is illustrated in figure $3 \mathrm{a}$, where the $M_{\mathrm{t}}$ and $M_{\mathrm{W}}$ measurements are compared to each other and to the SM calculation. Also here a preference for low values of $M_{\mathrm{H}}$ is visible.

Because the Higgs boson is not yet detected it is most interesting to determine which mass range is consistent with the current set of measurements. In the leading radiative correction terms the dependency on $M_{\hat{\mathrm{H}}}$ is only logarithmic. This weakens the indirect mass determination 


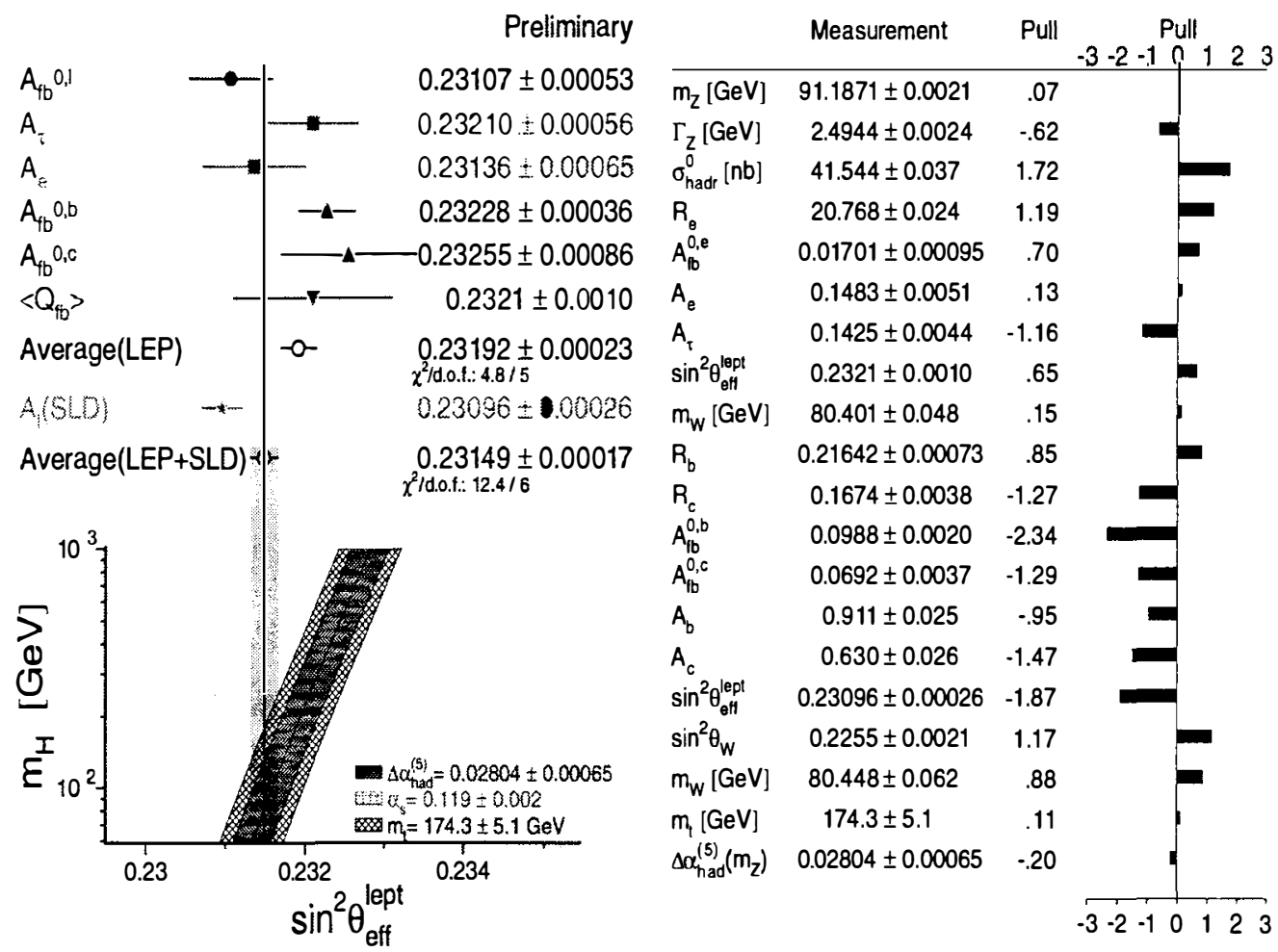

Figure 2: (a) The effective weak mixing angle, $\sin ^{2} \theta_{\text {eff }}^{\text {lept }}$, as determined in the measurements at LEP and SLD compared to the SM calculation for different Higgs masses. (b) All electroweak measurements which are input to the SM fits are listed including the pulls, which are the differences to the SM calculation in units of measurement error.

Table 3: Fit results for the SM parameters $M_{\mathrm{t}}, M_{\mathrm{H}}$ and $\alpha_{s}\left(M_{\mathrm{Z}}^{2}\right)$, from a combined analysis of different subsets of electroweak data. Also shown are the derived values of $\sin ^{2} \theta_{\mathrm{eff}}^{\text {lept }}, 1-M_{\mathrm{W}}^{2} / M Z^{2}$, and $M_{\mathrm{W}}$.

\begin{tabular}{|c|c|c|c|c|}
\hline & $\begin{array}{c}\text { LEP including } \\
\text { LEP } M_{\mathrm{W}}\end{array}$ & $\begin{array}{c}\text { all data except } \\
M_{\mathrm{W}} \text { and } M_{\mathrm{t}}\end{array}$ & $\begin{array}{c}\text { all data except } \\
M_{\mathrm{W}}\end{array}$ & all data \\
\hline \hline$M_{\mathrm{t}}[\mathrm{GeV}]$ & $176_{-11}^{+14}$ & $167_{-8}^{+10}$ & $172.9 \pm 4.7$ & $173.7 \pm 4.4$ \\
$M_{\mathrm{H}}[\mathrm{GeV}]$ & $147_{-92}^{+299}$ & $54_{-27}^{+81}$ & $78_{-41}^{+76}$ & $67_{-33}^{+60}$ \\
$\log \left(M_{\mathrm{H}} / \mathrm{GeV}\right)$ & $2.17_{-0.43}^{+0.48}$ & $1.73_{-0.30}^{+0.40}$ & $1.90_{-0.32}^{+0.29}$ & $1.82_{-0.30}^{+0.28}$ \\
$\alpha\left(M_{\mathrm{Z}}^{2}\right)$ & $0.120 \pm 0.003$ & $0.118 \pm 0.003$ & $0.118 \pm 0.003$ & $0.118 \pm 0.003$ \\
\hline$\chi^{2} /$ d.o.f. & $12 / 9$ & $22 / 12$ & $22 / 13$ & $23 / 15$ \\
\hline \hline $\sin ^{2} \theta_{\text {eft }}^{\text {lept }}$ & $0.23178 \pm 0.00021$ & $0.23151 \pm 0.00017$ & $0.23152 \pm 0.00017$ & $0.23145 \pm 0.00016$ \\
$1-M_{\mathrm{W}}^{2} / M_{\mathrm{Z}}^{2}$ & $0.2233 \pm 0.0006$ & $0.2232 \pm 0.0007$ & $0.2229 \pm 0.0005$ & $0.2227 \pm 0.0004$ \\
$M_{\mathrm{W}}[\mathrm{GeV}]$ & $80.363 \pm 0.030$ & $80.368 \pm 0.034$ & $80.382 \pm 0.026$ & $80.394 \pm 0.021$ \\
\hline
\end{tabular}



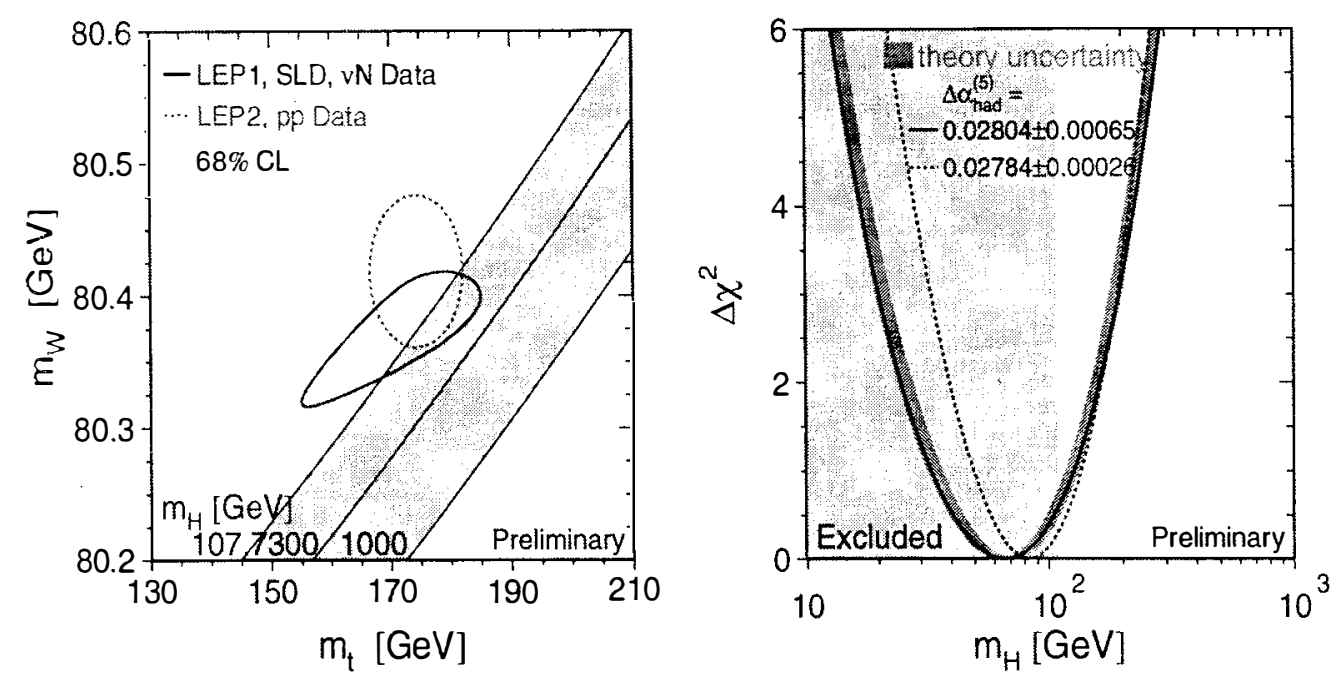

Figure 3: (a) Contour curves of the direct and indirect measurements of $M_{\mathrm{t}}$ and $M_{\mathrm{W}}$, compared to the SM predictions for different values of $M_{\mathrm{H}}$. (b) The figure shows the $\Delta \chi^{2}=\chi^{2}-\chi_{\min }^{2}$ dependence on $M_{\mathrm{H}}$ from a fit to all electroweak data. The band indicates the theoretical uncertainty due to higher order corrections. The dashed line is the calculation using a different value of $\Delta \alpha_{\text {had }}^{(5)}$. The Higgs mass region excluded by direct searches is marked by the grey area.

even when all electroweak measurements, many of them with permill accuracy, are used in the analysis. The result of the fit to the electroweak data is

$$
\log \left(M_{\mathrm{H}} / \mathrm{GeV}\right)=1.82_{-0.30}^{+0.28}, \quad M_{\mathrm{H}}=67_{-33}^{+60} \mathrm{GeV} .
$$

The low value is not a consequence of a single measurement, as can be seen in figures $2 \mathrm{a}$ and $3 \mathrm{a}$. The corresponding $\Delta \chi^{2}$-curve of the fit is shown in figure $3 \mathrm{~b}$. An upper limit on the Higgs mass can be set to

$$
M_{\mathrm{H}}<188 \mathrm{GeV} \text {, at } 95 \% \text { C.L. }
$$

But the central value and the error on $M_{\mathrm{H}}$ are strongly dependent on the value of $\Delta \alpha_{\mathrm{had}}^{(5)}$. With a value of $\Delta \alpha_{\text {had }}^{(5)}=0.02784 \pm 0.00026^{13}$ the error on $\log \left(M_{\mathrm{H}}\right)$ is reduced by $25 \%$ and the central value is shifted to $\log \left(M_{\mathrm{H}} / \mathrm{GeV}\right)=1.92$ or $M_{\mathrm{H}}=83.2 \mathrm{GeV}$, which is still on the low side of the mass spectrum. From direct searches at LEP values of $M_{\mathrm{H}}$ below $107.9 \mathrm{GeV}$ are excluded with $95 \%$ C.L. ${ }^{17}$, still compatible with the indirect result. However, the low value of $M_{\mathrm{H}}$ preferred by electroweak measurements give hope that a positive finding of the Higgs will come soon, either at LEP energies or by the Tevatron or the future LHC experiments.

\section{Acknowledgments}

I would like to thank the experiments ALEPH, DELPHI, L3, OPAL and SLD for making their most recent and preliminary results available. I especially like to thank the members of the LEP Electroweak Working Group and of the LEP WW Working Group for performing the combination of the individual measurements and for converting them into nice graphs and plots.

This work has been supported by the German Bundesministerium für Bildung und Forschung under the contract number $057 \mathrm{AC} 36 \mathrm{P} 3$. 


\section{References}

1. The LEP WW Working Group, Preprint, LEPEWWG/WW/00-01.

2. ALEPH Internal Note CONF 2000-015;

DELPHI Internal Note 99-64 CONF 251;

OPAL Internal Note PN 422;

L3 Internal Note 2520

3. LEP Energy Calibration Working Group, A. Blondel et al., Eur. Phys. J. C 11 (1999) 573 ; LEP Energy Calibration Working Group, Preprint, LEP ECAL/99-01

4. G. Wilkinson, Energy Calibration: Status and Prospects, Proceedings of the 10th Chamonix Workshop on LEP-SPS, Ed. P. Le Roux, et al., CERN-SL-2000-007.

5. G. Gustafson, U. Petterson and P.M. Zerwas, Phys. Lett. B 209 (1988) 90;

T. Sjöstrand and V.A. Khoze, Z. Phys. C 62 (1994) 281, Phys. Rev. Lett. 72 (1994) 28;

E. Accomando, A. Ballestrero and E. Maina, Phys. Lett. B 362 (1995) 141;

G. Gustaffson and J. Häkkinen, Z. Phys. C 64 (1994) 659;

L. Lönnblad, Z. Phys. C 70 (1996) 107;

J. Ellis and K. Geiger, Phys. Rev. D 54 (1996) 1967, Phys. Lett. B 404 (1997) 230.

6. K. Fialkowski and R. Wit, Z. Phys. C 74 (1997) 145; K. Fialkowski, R. Wit and J. Wosiek, Phys. Rev. D 58 (1998) 094013; S. Jadach and K. Zalewski, Acta. Phys. Pol. B 28 (1997) 1363; V. Kartvelishvili, R. Kvatadze and R. Møller, Phys. Lett. B 408 (1997) 331; L. Lönnblad and T. Sjöstrand, Phys. Lett. B 351 (1995) 293; L. Lönnblad and T. Sjöstrand, Eur. Phys. J. C 2 (1998) 165.

7. N. Neufeld, these proceedings.

8. U. Bassler, these proceedings.

9. The LEP and SLD Electroweak Working Groups, Preprint, CERN-EP/2000-016, and LEP EWWG contributions to the 2000 winter conferences; latest results are available from: http://www.cern.ch/LEPEWWG.

10. The CDF Collaboration, F. Abe et al., Phys. Rev. Let. 74 (1995) 341.

11. The Particle Data Group, C. Caso et al., Eur. Phys. J. C 3 (1999) 1.

12. S. Eidelmann and F. Jegerlehner, Z. Phys. C 67 (1995) 585.

13. M. Davier and A. Höcker, Phys. Lett. B 419 (1998) 419.

14. T. Wright, these proceedings.

15. R. Partridge, Heavy Quark Production and Decay ( $t$, $b$, and onia), talk presented at ICHEP'98, Vancouver, B.C., Canada, 23-29 July, 1998.

16. CCFR/NuTeV Collaboration, K. McFarland et al., Eur. Phys. Jour. C1 (1998) 509;

NuTeV Collaboration, K. McFarland, talk presented at the XXXIIIth Rencontres de Moriond, hep-ex/9806013.

17. E. Ferrer Ribas, these proceedings. 